\title{
Analysis of Mean Absolute Deviation for Randomized Block Design under Laplace Distribution
}

\author{
Elsayed A. H. Elamir \\ Department of Statistics and Mathematics, Benha University, Benha, Egypt \& Management \& Marketing Department, College of Business, \\ University of Bahrain, Manama, Kingdom of Bahrain
}

Email address:

shabib40@gmail.com

\section{To cite this article:}

Elsayed A. H. Elamir. Analysis of Mean Absolute Deviation for Randomized Block Design under Laplace Distribution. American Journal of Theoretical and Applied Statistics. Vol. 4, No. 3, 2015, pp. 138-149. doi: 10.11648/j.ajtas.20150403.19

\begin{abstract}
Analysis of mean absolute deviation (ANOMAD) for randomized block design is derived where the total sum of absolute deviation (TSA) is partition into exact block sum of absolute deviation (BLSA), exact treatment sum of absolute deviation (TRSA) and within sum of absolute deviation (WSA). The exact partitions are derived by getting rid of the absolute function from MAD by using the idea of re-expressing the mean absolute deviation as a weighted average of data with sum of weights zero. ANOMAD has advantages: offers meaningful measure of dispersion, does not square data, and can be extended to other location measures such as median. Two ANOMAD graphs are proposed. However, the variance-gamma distribution is used to fit the sampling distributions for the mean of BLSA and the mean of TRSA. Consequently, two tests of equal means and medians are proposed under the assumption of Laplace distribution.
\end{abstract}

Keywords: ANOVA, Effect Sizes, Laplace Distribution, MAD, Variance-Gamma Distribution

\section{Introduction}

There has been an increasing interest in properties and applications of a Laplace distribution (see, for example, [2], [12], [13], [10] and [23]. A Laplace distribution possesses a number of favourable characteristics which make it attractive for many applications; see, [12]. In particular, a simple closed form, stability and robustness to model misspecification. Laplace distribution is found to be especially appealing in modelling heavy tailed processes in finance, engineering, astronomy and environmental sciences ([9], [16], [15] and [21]. It may also offer certain pedagogical advantages; see, [1] and [7]. For extensive discussion and comparisons; see, [19] and [6].

A random variable has a Laplace distribution with location parameter $-\infty<\mu<\infty$ and scale $\Delta>0$ if its probability density function is

$$
f(y)=\frac{1}{2 \Delta} e^{-\frac{|y-\mu|}{\Delta}},-\infty<y<\infty
$$

The Laplace distribution has

$$
E(Y)=\mu, V(Y)=2 \Delta^{2} \text { and } M A D(Y)=\Delta
$$

The probability density function of the Laplace distribution is also reminiscent of the normal distribution; whereas the normal distribution is expressed in terms of the squared difference from the mean while the Laplace density is expressed in terms of the absolute difference from the mean or median.

A randomized complete block design is a restricted randomization design in which the experimental units are first sorted into homogeneous rows, called blocks, and the groups (treatments) are then assigned at random within the blocks; see, [17]. The model for a randomized complete block design containing the comparison of no interaction effects, when both the block and treatment effects are fixed and there are $B$ blocks (BL) and $G$ groups (TR), is as

$$
Y_{b g}=\mu+\rho_{b}+\tau_{g}+\varepsilon_{b g}
$$

$\mu$ is a constant, $\rho_{b}$ are constants for the block (row) effects, $\tau_{g}$ are constants for the group (column) effects and $\varepsilon_{b g}$ are independent $L(0, \Delta)$.

Analysis of mean absolute deviation (ANOMAD) for a randomized complete block design is derived where the total sum of absolute deviation (TSA) is partition into exact block sum of absolute deviation (BLSA), exact treatment sum of absolute deviation (TRSA) and within sum of absolute deviation (WSA). The exact partitions are derived by getting 
rid of the absolute function by using the idea of re-expressing the mean absolute deviation as a weighted average of data with sum of weights zero. Since the MAD is a natural parameter of the Laplace distribution, the sampling distributions of scaled BLSA and TRSA are studied under the assumption of Laplace distribution using the variance-gamma distribution (generalized Laplace distribution). Consequently, an analysis of mean absolute deviation is proposed to test for equal population means and medians and two measures of effect sizes are extended to ANOMAD test.

Representation of MAD as a weighted average is presented in Section 2. The partitions of TSA into exact BLSA, exact WSA and ANOMAD tables are derived in Section 3. The sampling distributions for block and treatment are introduced in Section 4. Two graphs are suggested in Section 5. ANOMAD tests for equal means and median with effect sizes are studied in Section 6. Section 7 is devoted to conclusion.

\section{Representation of MAD as a Weighted Average}

Let $Y_{1}, Y_{2}, \ldots, Y_{n}$ be a random sample from a continuous distribution with, density function $f(y)$, quantile function $y(F)=F^{-1}(y)=Q(F), 0<F<1$, cumulative distribution function $F(y)=F, \mu=E(Y)$ and $v=\operatorname{Med}(Y)$.

Elamir (2012) and [11] defined MAD about mean and median as

$$
\Delta_{\mu}=E|Y-\mu|=E\left[\left(2 I_{\mu}-2 \bar{I}_{\mu}\right) Y\right]
$$

and

$$
\Delta_{v}=E|Y-v|=E\left[\left(2 I_{v}-2 \bar{I}_{v}\right) Y\right]
$$

where

$$
I_{\mu}=\left\{\begin{array}{ll}
1, & Y>\mu \\
0, & Y \leq \mu
\end{array} \quad \text { and } \quad I_{v}= \begin{cases}1, & Y>v \\
0, & Y \leq v\end{cases}\right.
$$

From [5] this can be estimated as

$$
\widehat{\Delta}_{\mu}=\delta_{1}=\frac{1}{n} \sum_{i=1}^{n}\left(2 I_{\bar{y}}-2 \bar{I}_{\bar{y}}\right) y
$$

and

$$
\widehat{\Delta}_{v}=\delta_{2}=\frac{1}{n} \sum_{i=1}^{n}\left(2 I_{\tilde{y}}-2 \bar{I}_{\tilde{y}}\right) y
$$

where

$$
I_{\bar{y}}=\left\{\begin{array}{ll}
1, & y>\bar{y} \\
0, & y \leq \bar{y}
\end{array} \quad \text { and } \quad I_{\tilde{y}}= \begin{cases}1, & y>\tilde{y} \\
0, & y \leq \tilde{y}\end{cases}\right.
$$

\section{Exact MAD Partitions Mean and Median}

Assume there are $G$ different groups (treatments) with individuals in each group $y_{b g}$, with block $b=1,2, \ldots, B$, and $n=B G$. Let $y_{b g}-\bar{y}$ is the total deviation ( $\bar{y}=$ $\left.\sum_{g}^{G} \sum_{b}^{B} y_{b g} / n\right), \bar{y}_{g}-\bar{y}$ is the deviation of group mean $\left(\bar{y}_{g}=\sum_{b=1}^{B} y_{b g} / B\right)$ around total mean, $\bar{y}_{b}-\bar{y}$ is the deviation of block mean $\left(\bar{y}_{b}=\sum_{g=1}^{G} y_{b g} / G\right)$ around total mean and $y_{b g}-\bar{y}_{b}-\bar{y}_{g}+\bar{y}$ is the error or within.

The sample MAD about mean is

$$
\delta_{1}=\frac{1}{n} \sum_{i=1}^{n}\left(2 I_{\bar{y}}-2 \bar{I}_{\bar{y}}\right) y=\frac{1}{n} \sum_{i=1}^{n} w_{i} Y_{i}
$$

This is a weighted average form where

$$
w_{i}=\left(2 I_{i \bar{y}}-2 \bar{I}_{\bar{y}}\right)
$$

Note that,

$$
\sum_{i=1}^{n} w_{i}=0
$$

Therefore, the total sum of absolute value is considered to be

$$
T S A=\sum_{i=1}^{n}\left|Y_{i}-\bar{Y}\right|=\sum_{i=1}^{n} w_{i} Y_{i}
$$

This is the most important equation to obtain the exact analysis of mean absolute deviations about mean and median.

\subsection{Theorem 1}

In a randomized complete block design the total sum of absolute deviations about mean is partitions as

$$
T S A=B L S A+T R S A+W S A
$$

where

$$
\begin{gathered}
T S A=\sum_{g=1}^{G} \sum_{b=1}^{B}\left|Y_{b g}-\bar{Y}\right|=\sum_{g=1}^{G} \sum_{b=1}^{B} w_{b g}\left(Y_{b g}-\bar{Y}\right), \\
B L S A=\sum_{g=1}^{G} \sum_{b=1}^{B} w_{b g}\left(\bar{Y}_{b}-\bar{Y}\right) \\
T R S A=\sum_{g=1}^{G} \sum_{b=1}^{B} w_{b g}\left(\bar{Y}_{g}-\bar{Y}\right) \\
W S A=\sum_{g=1}^{G} \sum_{b=1}^{B} w_{b g}\left(Y_{b g}-\bar{Y}_{b}-\bar{Y}_{g}+\bar{Y}\right)
\end{gathered}
$$

and

$$
w_{b g}=\left(2 I_{b g}-2 \bar{I}_{\bar{y}}\right)
$$

Proof:

Where $\sum w=0$, the total sum of absolute deviations is 


$$
T S A=\sum_{i=1}^{n} w_{i} Y_{i}=\sum_{i=1}^{n} w_{i}\left(Y_{i}-\bar{Y}\right)
$$

By adding and subtracting $\bar{Y}_{b}, \bar{Y}_{g}$ and $\bar{Y}$ and taking the summation over both $g$ and $b$ then

$$
T S A=\sum_{g=1}^{G} \sum_{i=1}^{B} w_{b g}\left(Y_{b g}-\bar{Y}_{b}+\bar{Y}_{b}-\bar{Y}_{g}+\bar{Y}_{g}-\bar{Y}+\bar{Y}-\bar{Y}\right)
$$

Therefore,

$$
\begin{aligned}
& \sum_{g=1}^{G} \sum_{b=1}^{B} w_{b g}\left(Y_{b g}-\bar{Y}\right) \\
= & \sum_{g=1}^{G} \sum_{b=1}^{B} w_{b g}\left(\bar{Y}_{b}-\bar{Y}\right) \\
+ & \sum_{g=1}^{G} \sum_{b=1}^{B} w_{b g}\left(\bar{Y}_{g}-\bar{Y}\right) \\
+ & \sum_{g=1}^{G} \sum_{b=1}^{B} w_{b g}\left(Y_{b g}-\bar{Y}_{b}-\bar{Y}_{g}+\bar{Y}\right)
\end{aligned}
$$

\subsection{Theorem 2}

In a randomized complete block design the total sum of absolute deviations about median is partitions as

$$
T S A=B L S A+B S A+W S A
$$

where

$$
\begin{gathered}
T S A=\sum_{g=1}^{G} \sum_{b=1}^{B}\left|Y_{b g}-\tilde{Y}\right|=\sum_{g=1}^{G} \sum_{b=1}^{B} w_{b g}\left(Y_{b g}-\tilde{Y}\right), \\
B L S A=\sum_{g=1}^{G} \sum_{b=1}^{B} w_{b g}\left(\tilde{Y}_{b}-\check{Y}\right) \\
T R S A=\sum_{g=1}^{G} \sum_{b=1}^{B} w_{b g}\left(\tilde{Y}_{g}-\tilde{Y}\right) \\
W S A=\sum_{g=1}^{G} \sum_{b=1}^{B} w_{b g}\left(Y_{b g}-\tilde{Y}_{b}-\tilde{\tilde{Y}}_{g}+\tilde{Y}\right)
\end{gathered}
$$

and

$$
w_{b g}=\left(2 I_{b g}-2 \bar{I}_{\tilde{y}}\right)
$$

Proof: same as mean.

\subsection{Comparison with ANOVA}

The analysis of variance (ANOVA) for a randomized complete block design is

$$
\begin{aligned}
& \sum_{g=1}^{G} \sum_{b=1}^{B}\left(Y_{b g}-\bar{Y}\right)^{2} \\
= & \sum_{g=1}^{G} \sum_{b=1}^{B}\left(\bar{Y}_{b}-\bar{Y}\right)^{2}+\sum_{g=1}^{G} \sum_{b=1}^{B}\left(\bar{Y}_{g}-\bar{Y}\right)^{2} \\
+ & \sum_{g=1}^{G} \sum_{b=1}^{B}\left(Y_{b g}-\bar{Y}_{b}-\bar{Y}_{g}+\bar{Y}\right)^{2}
\end{aligned}
$$

See; for example, [17].

The analysis of mean absolute deviation (ANOMAD) for a randomized complete block design is

$$
\begin{aligned}
& \sum_{g=1}^{G} \sum_{b=1}^{B} w_{b g}\left(Y_{b g}-\bar{Y}\right) \\
= & \sum_{g=1}^{G} \sum_{b=1}^{B} w_{b g}\left(\bar{Y}_{b}-\bar{Y}\right) \\
+ & \sum_{g=1}^{G} \sum_{b=1}^{B} w_{b g}\left(\bar{Y}_{g}-\bar{Y}\right) \\
+ & \sum_{g=1}^{G} \sum_{b=1}^{B} w_{b g}\left(Y_{b g}-\bar{Y}_{b}-\bar{Y}_{g}+\bar{Y}\right)
\end{aligned}
$$

Note that:

1. ANOMAD replaces the square in ANOVA by weight and that ensures stability in statistical inferences.

2. ANOMAD can be extended to other measures of location easily, for example, median.

\subsection{Illustrative Example}

To have an idea on how the method work. Table 1 shows TSA partition for a hypothetical data. Note that, $T S A=57$, $B L S A=9, B S A=10$ and $W S A=38$ and the total $9+10+38=57$ that gives exact partitions

\subsection{Divisors and ANOMAD Tables}

ANOMAD is introduced and used to test for equal population means and medians under the following assumptions.

1. The observations are random and independent samples from the populations.

2. The distributions of the populations from which the samples are selected are Laplace distribution.

3. The $\Delta$ 's of the distributions in the populations are equal.

A simulation study is conducted to compute the suitable divisors for scaled BLSA, TRSA and WSA using the following steps:

1. For selected design simulate data from Laplace distribution using a very large number $N$.

2. Compute $B L S A / \Delta, T R S A / \Delta$, and $W S A / \Delta$ for each $G$ 
and $B$.

3. Compute the average for each one.

From the simulation results in Table 2, the proposed ANOMAD table about mean is summarized in Table 3. A simulation study is conducted to compute the suitable divisors for scaled BLSA, TRSA and WSA using the following steps:

1. For selected design simulate data from Laplace distribution using a very large number $N$.
2. Compute $B L S A / \Delta, T R S A / \Delta$, and $W S A / \Delta$ for each $G$ and $B$.

3. Compute the average for each one.

From the simulation results in Table 2, the proposed ANOMAD table about mean is summarized in Table 3. Also, from the simulation results in Table 2, the proposed ANOMAD table about median is summarized in Table 4.

Table 1. TSA partition into BLSA, TRSA and WSA for a hypothetical data.

\begin{tabular}{|c|c|c|c|c|c|c|c|}
\hline$y$ & $|y-\bar{y}|$ & $I$ & $w$ & $w y$ & $w\left(\bar{y}_{b}-\bar{y}\right)$ & $w\left(\bar{y}_{g}-\bar{y}\right)$ & $w\left(y-\bar{y}_{g}-\bar{y}_{b}+\bar{y}\right)$ \\
\hline \multicolumn{8}{|c|}{ Group 1} \\
\hline 8 & 0.5 & 1 & 1.167 & 9.336 & 2.917 & 2.917 & -5.25 \\
\hline 11 & 3.5 & 1 & 1.167 & 12.837 & -0.583 & 2.917 & 1.75 \\
\hline 18 & 10.5 & 1 & 1.167 & 21.006 & 0.583 & 2.917 & 8.75 \\
\hline 3 & 4.5 & 0 & -0.833 & -2.499 & 2.083 & -2.082 & 3.75 \\
\hline \multicolumn{8}{|c|}{ Group 2} \\
\hline 20 & 12.5 & 1 & 1.167 & 23.34 & 2.917 & 0 & 11.67 \\
\hline 1 & 6.5 & 0 & -0.833 & -0.833 & 0.417 & 0 & 5.00 \\
\hline 4 & 3.5 & 0 & -0.833 & -3.332 & -0.417 & 0 & 3.33 \\
\hline 5 & 2.5 & 0 & -0.833 & -4.165 & 2.083 & 0 & 0.00 \\
\hline \multicolumn{8}{|c|}{ Group 3} \\
\hline 2 & 5.5 & 0 & -0.833 & -1.666 & -2.083 & 2.082 & 4.58 \\
\hline 9 & 1.5 & 1 & 1.167 & 10.503 & -0.583 & -2.917 & 5.25 \\
\hline 2 & 5.5 & 0 & -0.833 & -1.666 & -0.417 & 2.082 & 2.91 \\
\hline 7 & 0.5 & 0 & -0.833 & -5.831 & 2.083 & 2.082 & -3.75 \\
\hline Total & 57 & & & 57 & 9 & 10 & 38 \\
\hline
\end{tabular}

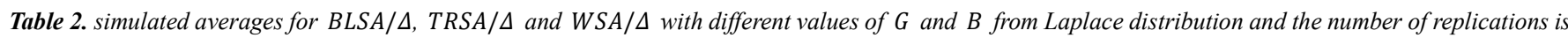
10000 .

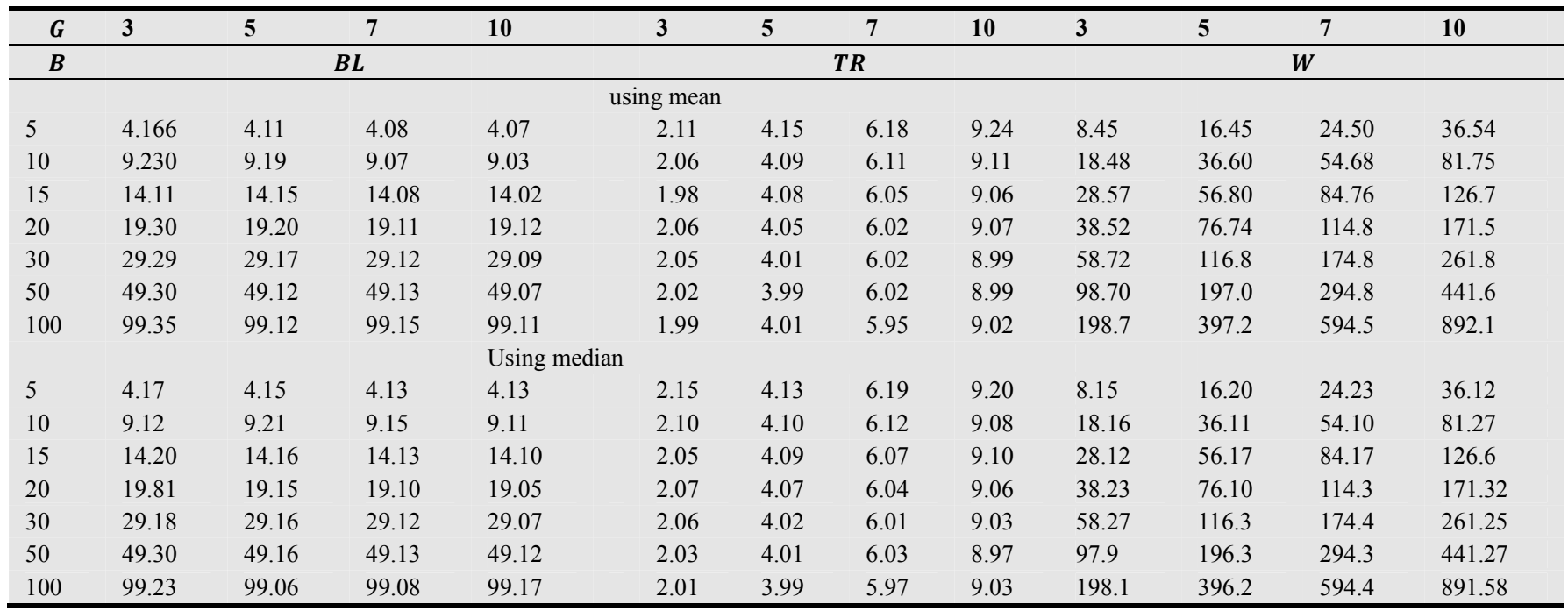

Table 3. summary of ANOMAD table about mean

\begin{tabular}{llll}
\hline Variation & Sum of absolute & Divisor & MAD estimate \\
\hline Block & $B L S A$ & $d 1=(B-1)+\frac{1}{G}$ & $M B L=\frac{B L S A}{d 1}$ \\
Treatment & $T R S A$ & $d 2=(G-1)+\frac{1}{B}$ & $M T R=\frac{T R S A}{d 2}$ \\
Within & $W S A$ & $d 3=(B-1)(G-1)+\frac{G+B}{G B}$ & $M W=\frac{W S A}{d 3}$ \\
Total & $T S A$ & & $R_{1}=\frac{M B L}{M W}$ \\
\hline
\end{tabular}

Table 4. summary of ANOMD table about median.

\begin{tabular}{llll}
\hline Variation & Sum of absolute & Divisor & MAD estimate \\
\hline Block & $B L S A$ & $d 1=(B-1)+\frac{1}{G}$ & $M B L=\frac{B L S A}{d 1}$ \\
Treatment & $T R S A$ & $d 2=(G-1)+\frac{1}{B}$ & $M T R=\frac{T R S A}{d 2}$ \\
Within & $W S A$ & $d 3=(B-1)(G-1)+\frac{G+B}{G B}$ & $M W=\frac{W S A}{d 3}$ \\
Total & $T S A$ & & $R_{3}=\frac{M B L}{M W}$ \\
\hline
\end{tabular}


Table 5. simulated critical right tail values for $R_{1}$ and $R_{2}$ using Laplace distribution for different $B$ and $G$ and the number of replications is 10000 .

\begin{tabular}{|c|c|c|c|c|c|c|c|c|c|c|}
\hline \multicolumn{11}{|c|}{$1-\alpha=0.95$} \\
\hline$G$ & 3 & 4 & 5 & 8 & 10 & 3 & 4 & 5 & 8 & 10 \\
\hline$B$ & \multicolumn{5}{|c|}{$R_{1}$} & \multicolumn{5}{|c|}{$R_{2}$} \\
\hline 10 & 3.01 & 2.58 & 2.50 & 2.26 & 2.25 & 4.41 & 3.46 & 3.08 & 2.48 & 2.25 \\
\hline 20 & 2.12 & 2.00 & 1.92 & 1.83 & 1.77 & 3.78 & 3.15 & 2.81 & 2.31 & 2.15 \\
\hline 25 & 1.99 & 1.86 & 1.78 & 1.73 & 1.70 & 3.76 & 3.10 & 2.81 & 2.29 & 2.14 \\
\hline 30 & 1.84 & 1.76 & 1.70 & 1.65 & 1.64 & 3.73 & 3.08 & 2.78 & 2.28 & 2.11 \\
\hline 50 & 1.61 & 1.55 & 1.51 & 1.48 & 1.48 & 3.54 & 3.00 & 2.75 & 2.21 & 2.10 \\
\hline 100 & 1.40 & 1.38 & 1.34 & 1.33 & 1.32 & 3.42 & 2.99 & 2.73 & 2.20 & 2.10 \\
\hline \multicolumn{11}{|c|}{$1-\alpha=0.99$} \\
\hline 10 & 4.25 & 3.93 & 3.60 & 3.13 & 3.18 & 7.50 & 6.20 & 4.77 & 3.50 & 3.07 \\
\hline 15 & 3.94 & 3.17 & 2.80 & 2.63 & 2.49 & 6.99 & 5.24 & 4.40 & 3.28 & 2.88 \\
\hline 20 & 2.87 & 2.64 & 2.48 & 2.26 & 2.23 & 6.34 & 4.81 & 4.22 & 3.20 & 2.85 \\
\hline 25 & 3.00 & 2.35 & 2.25 & 2.14 & 2.08 & 6.23 & 4.74 & 4.15 & 3.10 & 2.84 \\
\hline 30 & 2.33 & 2.19 & 2.17 & 2.00 & 2.00 & 5.83 & 4.84 & 4.14 & 3.10 & 2.82 \\
\hline 50 & 1.96 & 1.88 & 1.80 & 1.74 & 1.72 & 5.65 & 4.65 & 4.05 & 3.07 & 2.77 \\
\hline 100 & 1.59 & 1.58 & 1.53 & 1.49 & 1.48 & 5.53 & 4.45 & 3.98 & 3.06 & 2.72 \\
\hline
\end{tabular}

Table 6. simulated critical right tail values for $R_{3}$ and $R_{4}$ using Laplace distribution for different $B$ and $G$ and the number of replications is 10000 .

\begin{tabular}{|c|c|c|c|c|c|c|c|c|c|c|}
\hline \multicolumn{11}{|c|}{$1-\alpha=0.95$} \\
\hline$G$ & 3 & 4 & 5 & 8 & 10 & 3 & 4 & 5 & 8 & 10 \\
\hline$B$ & \multicolumn{5}{|c|}{$R_{3}$} & \multicolumn{5}{|c|}{$\boldsymbol{R}_{4}$} \\
\hline 10 & 2.73 & 2.36 & 2.27 & 2.04 & 2.02 & 3.79 & 3.02 & 2.69 & 2.17 & 2.02 \\
\hline 20 & 1.96 & 1.81 & 1.76 & 1.67 & 1.64 & 3.31 & 2.79 & 2.51 & 2.10 & 1.94 \\
\hline 25 & 1.79 & 1.70 & 1.67 & 1.58 & 1.56 & 3.29 & 2.70 & 2.50 & 2.06 & 1.94 \\
\hline 30 & 1.70 & 1.64 & 1.58 & 1.52 & 1.52 & 3.25 & 2.69 & 2.48 & 2.05 & 1.91 \\
\hline 50 & 1.51 & 1.45 & 1.43 & 1.39 & 1.37 & 3.12 & 2.65 & 2.43 & 2.03 & 1.91 \\
\hline 100 & 1.33 & 1.31 & 1.29 & 1.26 & 1.25 & 3.03 & 2.62 & 2.42 & 2.02 & 1.90 \\
\hline \multicolumn{11}{|c|}{$1-\alpha=0.99$} \\
\hline 10 & 3.99 & 3.36 & 3.28 & 2.74 & 2.72 & 6.35 & 4.79 & 4.21 & 3.08 & 2.64 \\
\hline 15 & 3.12 & 2.68 & 2.56 & 2.32 & 2.28 & 5.90 & 4.64 & 3.99 & 2.84 & 2.61 \\
\hline 20 & 2.69 & 2.42 & 2.20 & 2.08 & 2.02 & 5.40 & 4.37 & 3.61 & 2.83 & 2.56 \\
\hline 25 & 2.38 & 2.15 & 2.07 & 1.91 & 1.89 & 5.24 & 4.11 & 3.50 & 2.77 & 2.51 \\
\hline 30 & 2.19 & 2.00 & 1.95 & 1.81 & 1.78 & 5.23 & 4.06 & 3.49 & 2.74 & 2.46 \\
\hline 50 & 1.81 & 1.72 & 1.65 & 1.60 & 1.60 & 4.82 & 3.87 & 3.48 & 2.72 & 2.46 \\
\hline 100 & 1.49 & 1.47 & 1.44 & 1.40 & 1.40 & 4.77 & 3.85 & 3.47 & 2.69 & 2.45 \\
\hline
\end{tabular}

\section{Fitting Sampling Distributions}

Two approaches are used to obtain the approximations of the sampling distributions for $R_{1}, R_{2}, R_{3}$, and $R_{4}$.

\subsection{Simulation Approach}

The following steps are used to obtain the critical values:

1. For any given design simulate data from Laplace distribution using large number $N$.

2. Compute $R$ for each $N$.

3. Use quantile function in software $\mathrm{R}$ to obtain the required quantile for $R$.

Tables 5 and 6 give the simulated critical right tail values for $R_{1}, R_{2}, R_{3}$ and $R_{4}$ based on Laplace distribution for different $B$ and $G$ for $\alpha=0.05$ and 0.01 ..

\subsection{Variance Gamma Approach}

The random variable $Y$ is said to have Variance-Gamma (VG) with parameters $c, \theta \in R, v, \sigma>0$, if it has probability density function given by

$$
f(y ; c, \sigma, \theta, v)=\left(t_{1} / t_{2}\right)\left(t_{3} / t_{4}\right)^{a} K_{b}\left[\left(t_{5} / t_{6}\right)\right], y \in R
$$

where

$$
\begin{gathered}
t_{1}=2 \exp \left(\theta(y-c) / \sigma^{2}\right), t_{2}=\sigma \sqrt{2 \pi} v^{\frac{1}{v}} \Gamma(1 / v) \\
t_{3}=|y-c|, t_{4}=\sqrt{\left(2 \sigma^{2} / v+\theta^{2}\right)} \\
a=1 / v-1, b=1 / v-1 / 2 \\
t_{5}=t_{3} t_{4} / \sigma^{2}, t_{2}=\sigma \sqrt{2 \pi} v^{\frac{1}{v}} \Gamma(1 / v)
\end{gathered}
$$

Where $K_{v}(x)$ is a modified Bessel function of the third kind; see, for example, [22], and [8].

Note that there are other versions of this distribution available but this version is chosen because there is a software package in $\mathrm{R}$ called gamma-variance based on this version. The first two moments of this distribution are used to obtain a suitable fit for $R_{1}, R_{2}, R_{3}$ and $R_{4}$. The first two moments are

$$
E(Y)=c+\theta \text { and } V(Y)=\sigma^{2}+v \theta^{2}
$$

This distribution is defined over the real line and has many distributions as special cases or limiting distributions such as Gamma distribution in the limit $\sigma \downarrow 0$ and $c=0$, Laplace distribution as $\theta=0$ and $v=2$ and normal distribution as $\theta=0, v=1 / r$ and $r \rightarrow \infty$. By using the variances of $R_{1}, R_{2}, R_{3}$ and $R_{4}$ in Table 7 a very good fitting could be 
obtained. This fitting is given in Table 8 .

Table 7. simulated variances for $R_{1}, R_{2}, R_{3}$ and $R_{4}$ with different values of $G$ and $B$ from Laplace distribution and the number of replications is 10000 .

\begin{tabular}{|c|c|c|c|c|c|c|c|c|}
\hline$G$ & 3 & 5 & 7 & 10 & 3 & 5 & 7 & 10 \\
\hline$B$ & \multicolumn{5}{|c|}{$R_{1}$} & \multicolumn{3}{|c|}{$R_{2}$} \\
\hline 10 & 1.132 & 0.572 & 0.417 & 0.410 & 3.482 & 1.124 & 0.629 & 0.391 \\
\hline 20 & 0.313 & 0.213 & 0.198 & 0.182 & 1.997 & 0.831 & 0.576 & 0.356 \\
\hline 30 & 0.188 & 0.141 & 0.124 & 0.120 & 1.816 & 0.800 & 0.545 & 0.345 \\
\hline 50 & 0.100 & 0.081 & 0.073 & 0.070 & 1.662 & 0.761 & 0.509 & 0.344 \\
\hline 100 & 0.048 & 0.039 & 0.035 & 0.034 & 1.570 & 0.777 & 0.505 & 0.352 \\
\hline 10 & 0893 & 0377 & ${ }^{R}$ & \multicolumn{5}{|c|}{$R_{4}$} \\
\hline 20 & $\begin{array}{l}0.893 \\
0.270\end{array}$ & 0.152 & $\begin{array}{l}0.319 \\
0.139\end{array}$ & $\begin{array}{l}0.292 \\
0.123\end{array}$ & $\begin{array}{l}.496 \\
1.473\end{array}$ & $\begin{array}{l}0.151 \\
0.601\end{array}$ & $\begin{array}{l}0.445 \\
0.396\end{array}$ & $\begin{array}{l}0.2 / 4 \\
0.245\end{array}$ \\
\hline 30 & 0.135 & 0.098 & 0.880 & 0.079 & 1.253 & 0.556 & 0.361 & 0.237 \\
\hline 50 & 0.071 & 0.056 & 0.051 & 0.046 & 1.129 & 0.527 & 0.361 & 0.235 \\
\hline 100 & 0.034 & 0.027 & 0.025 & 0.023 & 1.044 & 0.522 & 0.350 & 0.237 \\
\hline
\end{tabular}

Table 8. variance gamma distribution approximation to ratio $R_{1}, R_{2}, R_{3}$ and $R_{4}$ by using the simulated first two moments.

\begin{tabular}{ll}
\hline Ratio & Variance gamma fitting \\
\hline$R_{1}$ & $V G\left(c=0, \sigma=\sqrt{\frac{2(B-1)}{[(B-1)(G-1)]^{2}}+\frac{1}{(B-1)}}, \theta=1, v=\frac{2.5}{(B-1)}\right)$ \\
$R_{2}$ & $V G\left(c=0, \sigma=\sqrt{\frac{2(G-1)}{[(B-1)(G-1)]^{2}}+\frac{1}{(G-1)}}, \theta=1, v=\frac{2.5}{(G-1)}\right)$ \\
$R_{3}$ & $V G\left(c=0, \sigma=\sqrt{\frac{n}{[(B-1)(G-1)]^{2}+1 / G}}, \theta=1, v=\frac{2.25}{(B-1)}\right)$ \\
$R_{4}$ & $V G\left(c=0, \sigma=\sqrt{\frac{n}{[(B-1)(G-1)]^{2}+1 / B}}, \theta=1, v=\frac{2.25}{(G-1)}\right)$ \\
\hline
\end{tabular}

(a)

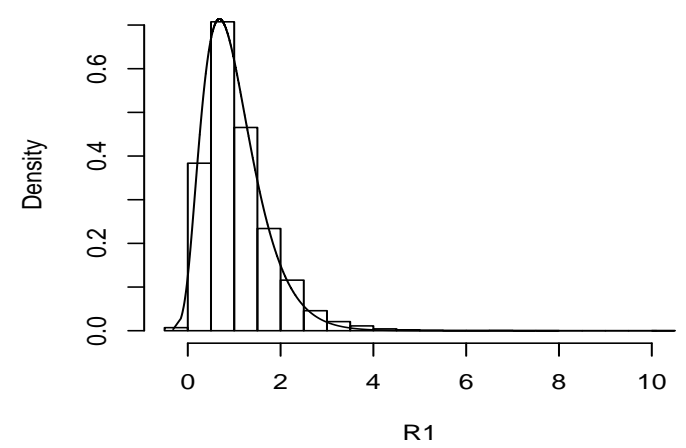

(c)

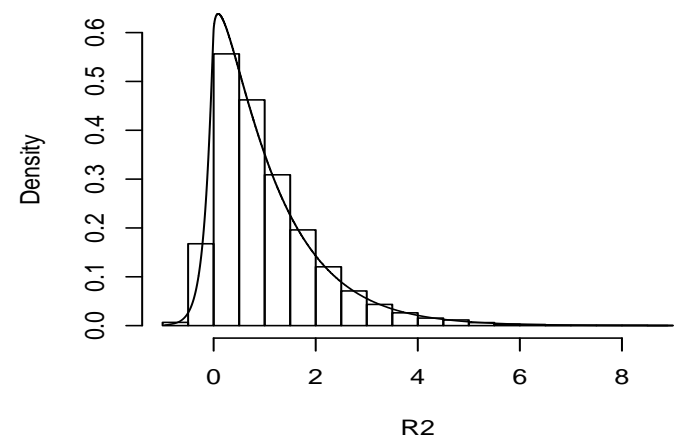

(b)

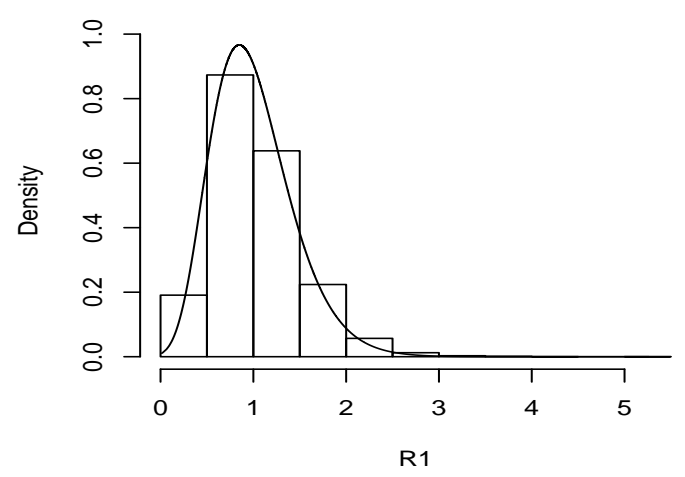

(d)

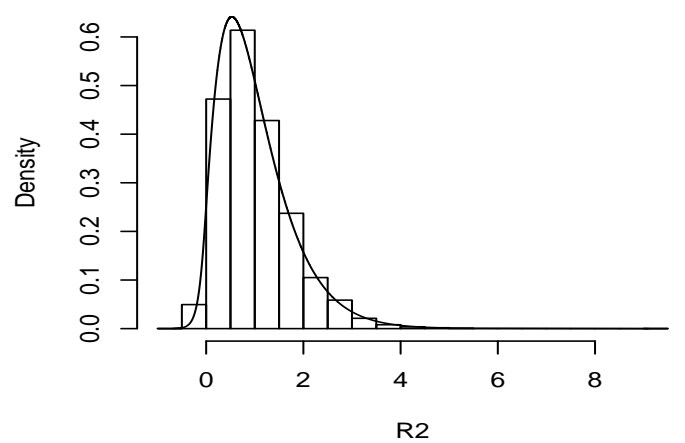

Figure 1. histogram of $R_{1}$ and $R_{2}$ based on simulated data from Laplace distribution with VG distribution superimposed and (a) $G=5$ and $B=10$ (b) $G=5$ and $B=20$ (c) $G=4$ and $B=25$ and (d) $G=7$ and $B=25$. 
(a)

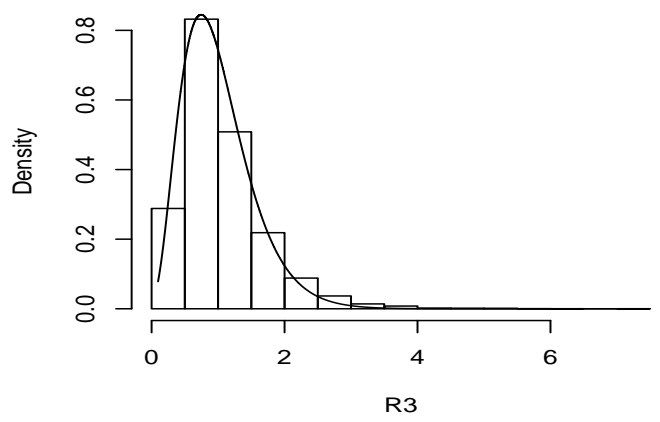

(c)

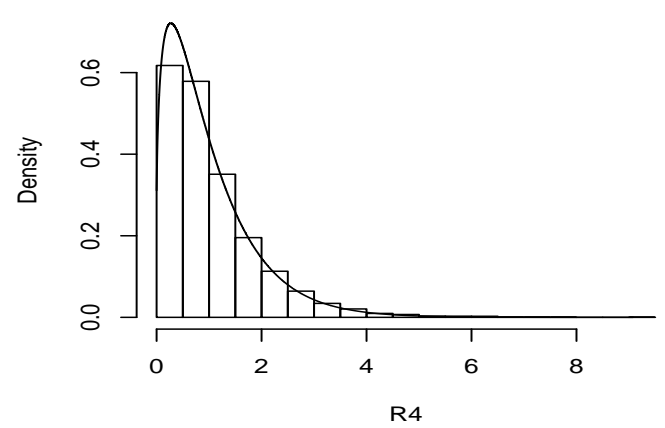

(b)

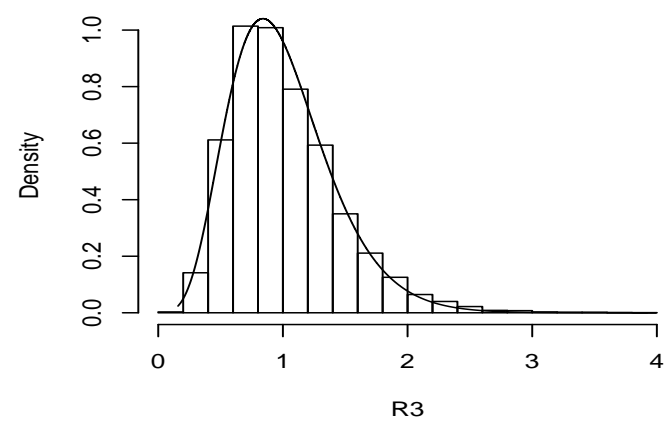

(d)

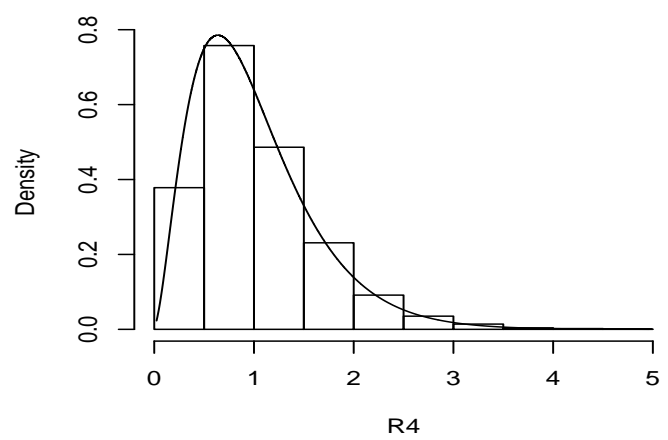

Figure 2. histogram of $R_{3}$ and $R_{4}$ based on simulated data from Laplace distribution with VG distribution superimposed and (a) $G=5$ and $B=10$ (b) $G=7$ and $B=15$ (c) $G=4$ and $B=20$ and (d) $G=7$ and $B=25$.

Figures 1 and 2 show the histograms of $R_{1}, R_{2}, R_{3}$ and $R_{4}$ using simulated data from Laplace distribution with VG distribution superimposed for different values of $G$ and $n$. It is clear that the variance gamma distribution gives a very good fit for the ratios.

\section{Graphic Presentation}

\subsection{ANOMAD General Plot}

This plot is for all groups to detect shifts in mean or median. The $X$-axis contains the index of the groups and the $Y$-axis contains the heights for the sum of $h_{1}=w\left(\bar{y}_{g}-\right.$ $\bar{y}) /((g-1) \Delta), \quad$ and $\quad h_{2}=w\left(y-\bar{y}_{g}-\bar{y}_{b}+\bar{y}\right) /((b-$ 1) $(g-1)) \Delta)$ for each group. Separate curves are drawn for sums of $h_{1}$ and $h_{2}$. The points on each curve are connected by lines. This graph should reflect the heights, shifts, and patterns among all groups.

\subsection{ANOMAD Individual Plot}

This plot is for each group to detect shift inside the group. The $X$-axis contains the index of the data for each group $\left(1,2, \ldots, n_{g}, g=1,2, \ldots, G\right)$ and the $Y$-axis contains the heights, $\quad h_{3}=w\left(\bar{y}_{g}-\bar{y}\right) /((g-1) \Delta) \quad$ and $\quad h_{4}=w(y-$ $\left.\bar{y}_{g}-\bar{y}_{b}+\bar{y}\right) /((b-1)(g-1)) \Delta$ for each value. Separate curves are drawn $h_{3}$ and $h_{4}$. The points on each curve are connected by lines. This graph should reflect the heights, shifts and patterns in each group.

Figures 3, 4 and 5 show that:

1. When means or medians are equals the two lines will be near from each other and most likely that there will be interference among them or the treatment line may be down the within line; see, Figure 3 a0. In this case it will not be clear pattern in each group and the heights will be almost the same for on each group; see, Figure 3 a1, a2, a3, a4 and a5. This may be indicating a strong evidence for no shifting in means or medians.

2. When mean(s) or median(s) are not equals the treatment line will start to go up until it may be separated from the within line; see, Figure 5 a0. In this case it will be clear pattern in group(s) with clear different gaps or heights; see, Figue $5 \mathrm{a} 1, \mathrm{a} 2, \mathrm{a} 3, \mathrm{a} 4$ and a5. It is clear that the group three has a different pattern from others. This may give a strong evidence for shift(s) in mean(s) or median(s).

3. If much more points of between line lies under within line with small gap; see, Figure $4 \mathrm{a} 0$. In this case there is not enough evidence for shift and it will not be clear pattern in each group; see, Figure 4 a1, a2, a3, a4 and a5. 

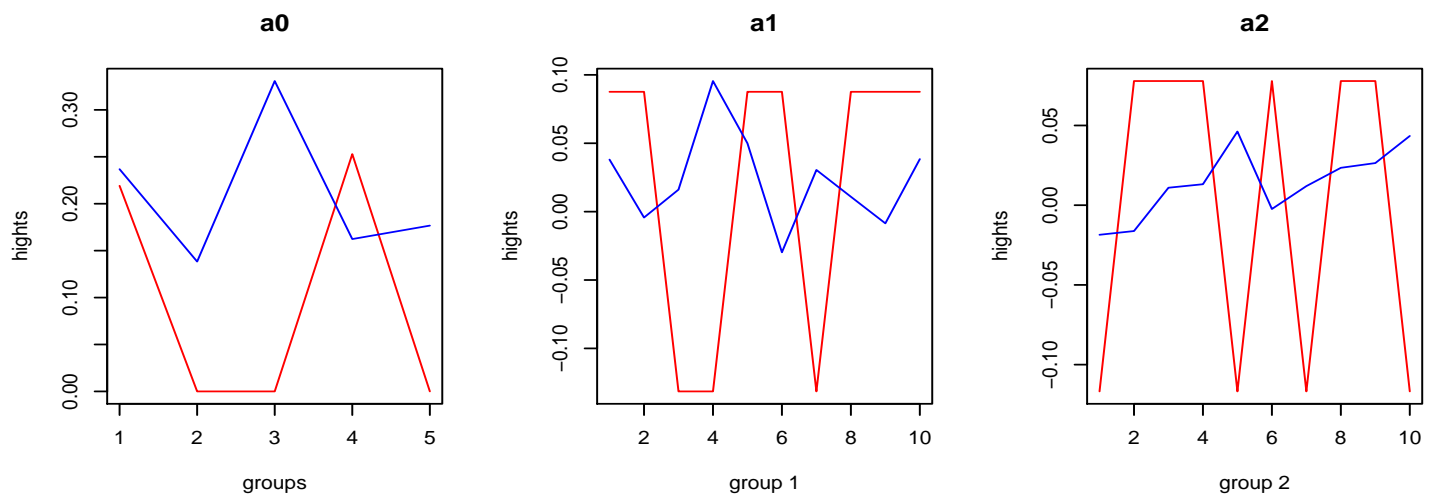

a3

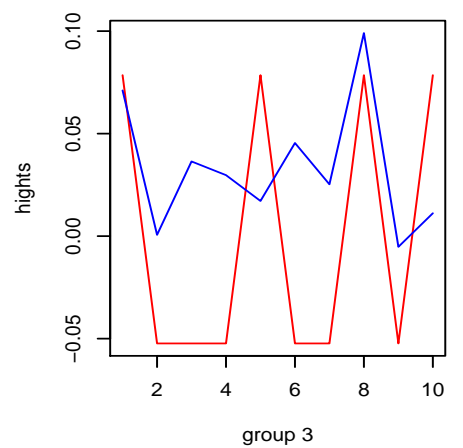

a4
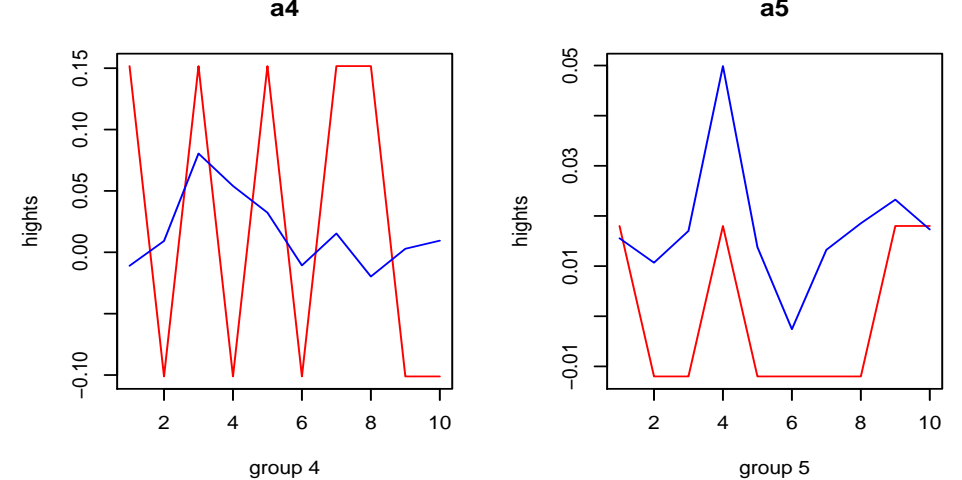

Figure 3. ANOMAD plot for simulated data from L(10,1): (a0) all groups and (a1), (a2), (a3), (a4) and (a5) for each group and $G=5$, $n=50$. Red line is treatment and blue line is within.
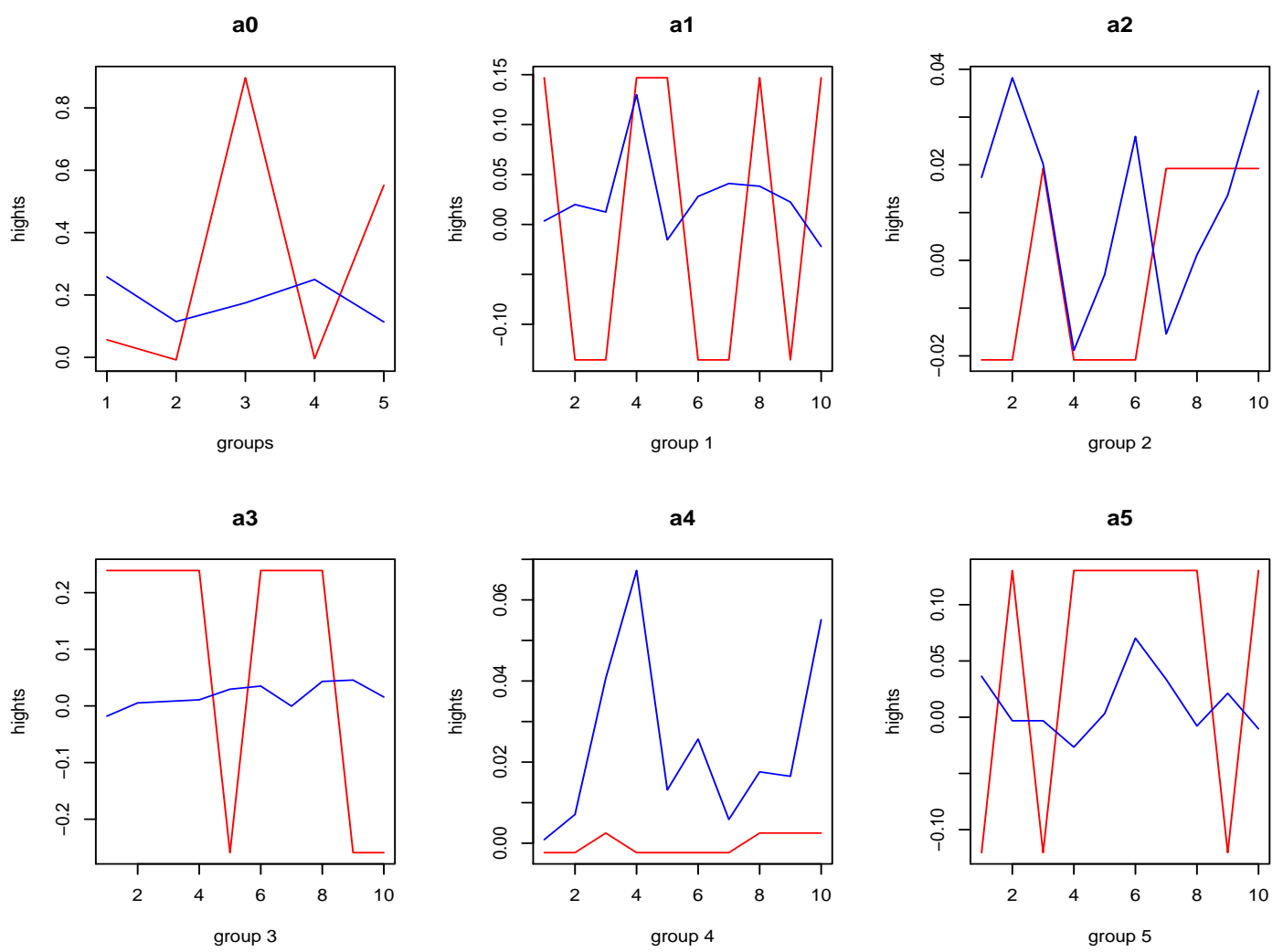

Figure 4. ANOMAD plot for simulated four groups $L(10,1)$ and one group L(11,1): (a0) all groups and (a1), (a2), (a3), (a4) and (a5) for each group and $G=5$, $n=50$. Red line is treatment and blue line is within. 


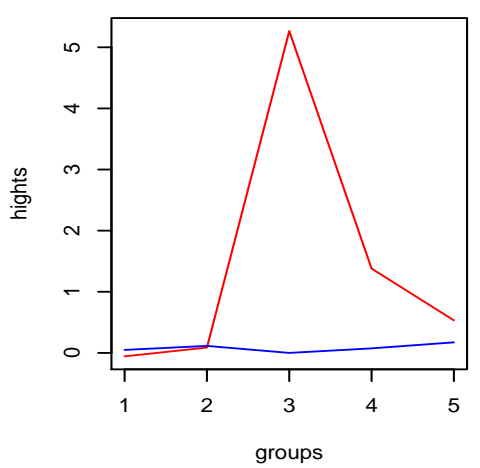

a3

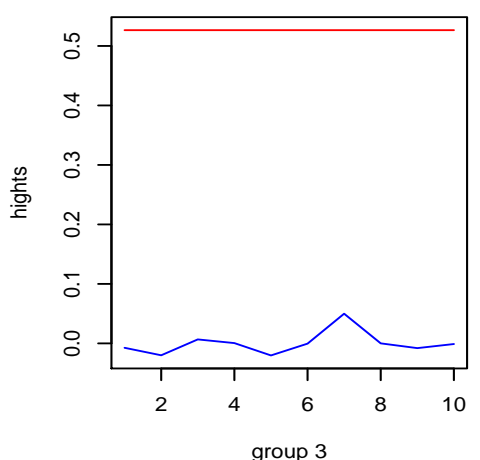

a1

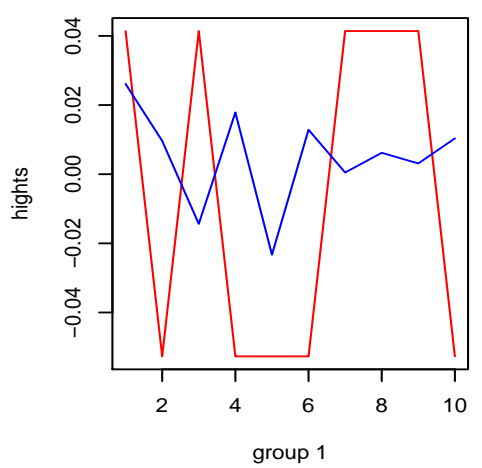

a4

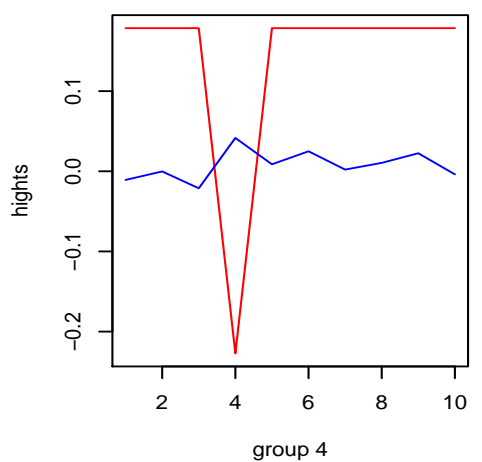

a2

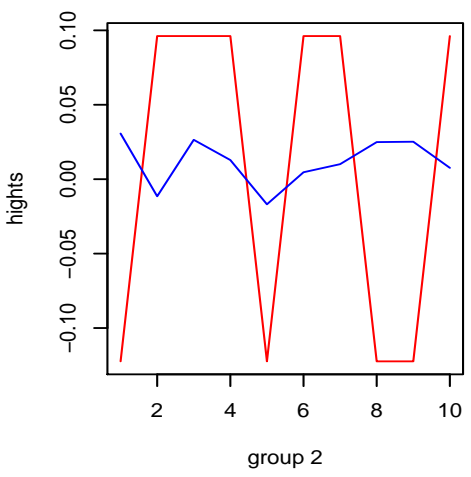

a5

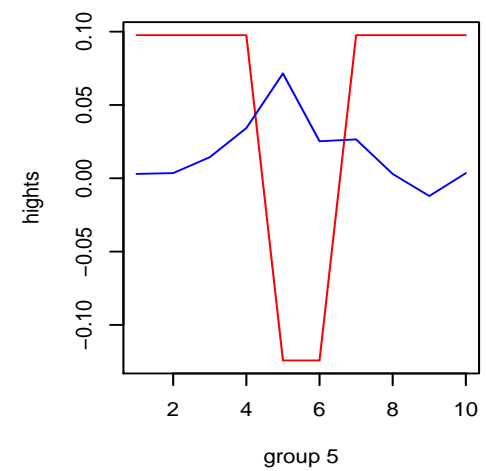

Figure 5. ANOMAD plot for simulated four groups $L(10,1)$ and one group L(11,1): (a0) all groups and (a1), (a2), (a3), (a4) and (a5) for each group and $G=5$, $n=50$. Red line is treatment and blue line is within.

\section{Test for mean and median}

For mean the null hypothesis $H_{0}$ is that Blocks

$$
H_{0}: \mu_{1}=\mu_{2}=\mu_{3}=\cdots=\mu_{B}
$$

Treatments

$$
H_{0}: \mu_{1}=\mu_{2}=\mu_{3}=\cdots=\mu_{G}
$$

For median the null hypothesis is that Blocks

$$
H_{0}: v_{1}=v_{2}=v_{3}=\cdots=v_{B}
$$

Treatments

$$
H_{0}: v_{1}=v_{2}=v_{3}=\cdots=v_{G}
$$

Bukhari is a food chain with four outlets in Bahrain. The owner is interested in determine if the average service quality at the four outlets is the same. Twelve people are selected and they asked to eat at each of the four outlets. The order of visits to the four outlets was randomized, but each customer visited each outlet one time. After each visit, each customer rated the service on a scale of 1 to 100. The data is given in Table 9 .

To test for the assumption of Laplace distribution, the function laplace.test() in package lawstat in R-software is used where it gives five goodness of fit for the Laplace distribution based on the work of [20]. Table 9 gives the sample data with means, medians, MAD and Kolmogorov-Smirnov (D) test for Laplace distribution. The results for the four groups are given in Table 9 where $p$-values more than $0.01,0.05$ and 0.10 , therefore, the assumption of Laplace cannot be rejected. Because the maximum MAD to minimum MAD is 1.3 , the assumption of homogeneity of MAD's may not be rejected.

From Table 10 , since $R_{1}=1.9<2.10$ the null hypothesis could not be rejected, i.e., blocking is not effective while $R_{2}=6.2>3.14$, therefore, the outlets are different in averages.

Figure 6 shows that

1. Most of the points of treatment line are above the within line with a big gap at the point 3 . This might give a visual evidence of shift in average for group 3; see, Figure $6 \mathrm{a} 0$.

2. The second group is stable while the first and fourth groups almost have the same patterns. The third group has pattern near from first and fourth groups but the gap is much more than the other groups. This may indicate that the third group is different from others; see, Figure 6 a1, a2, a3 and a4.

3. Table 11 shows that the block and treatment are significance in terms of equal medians. Care needs to be used in interpreting the implication of block effects. 
a0

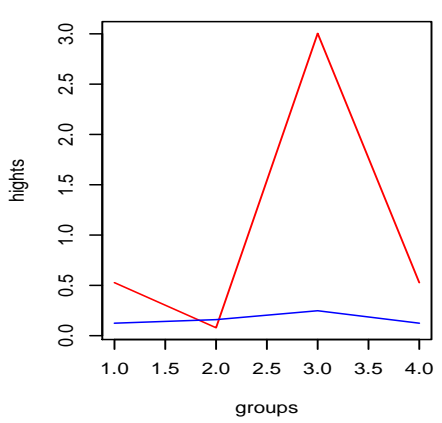

a0

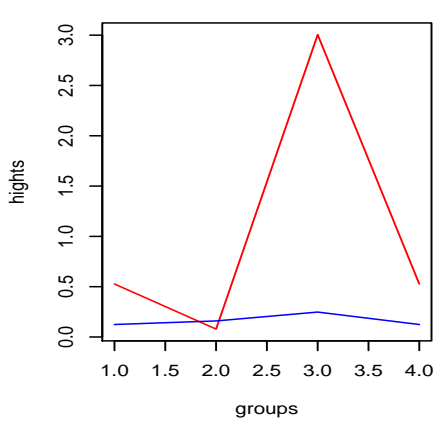

a1

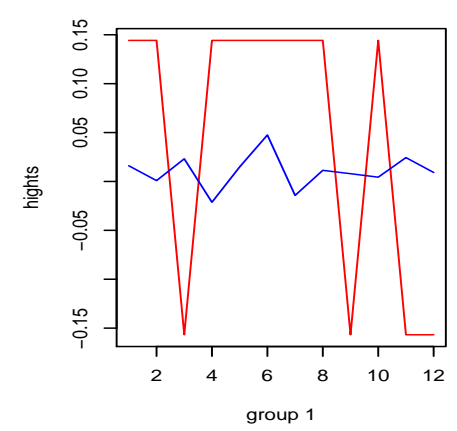

a3

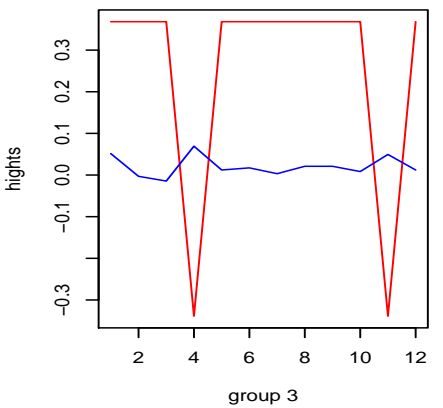

a2

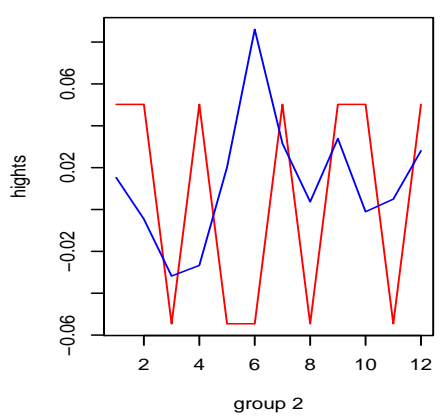

a4

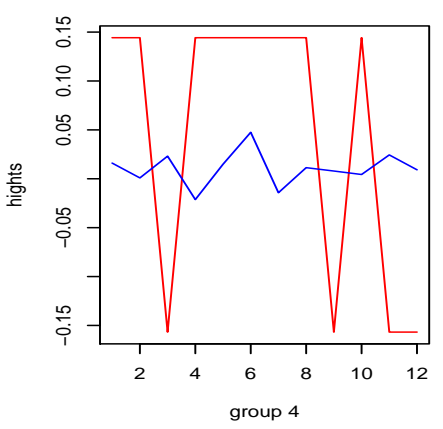

Figure 6. ANOMAD plots for the service quality data for four outlets.

Table 9. service quality score for four outlets and Laplace goodness of fit using Kolmogorov-Smirnov (KS) test.

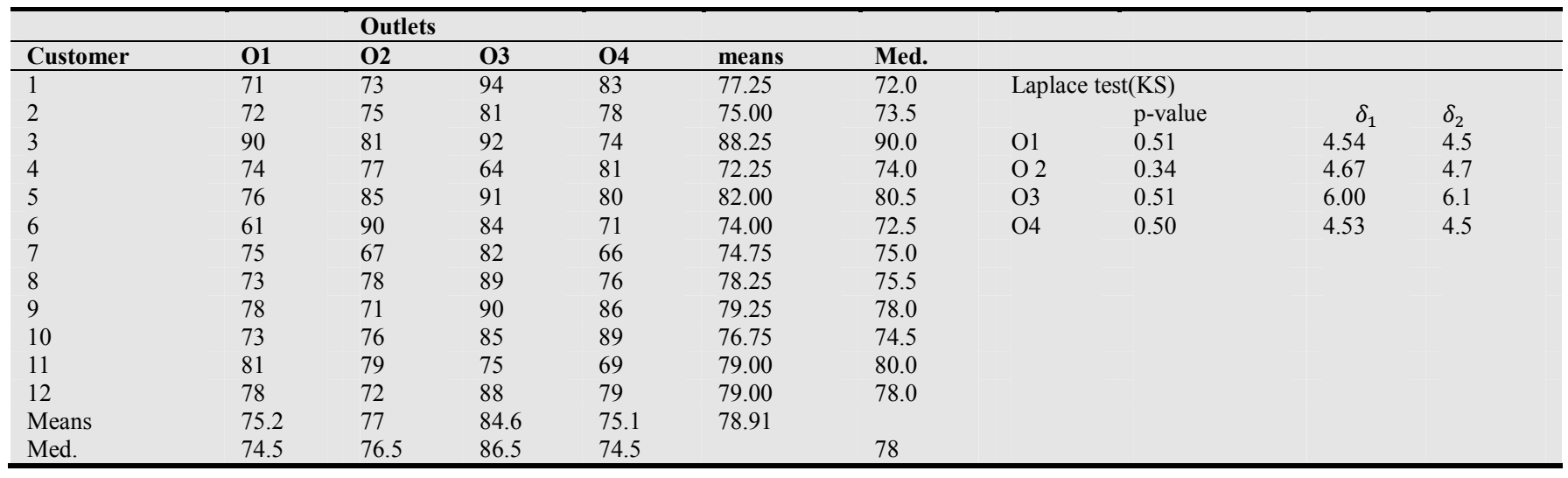

Table 10. ANOMAD for testing equal means for quality service.

\begin{tabular}{llllrl}
\hline Varia. & SA & Divisor & MAD est. & $\boldsymbol{R}$ & $\boldsymbol{q} \boldsymbol{V} \boldsymbol{G}_{\mathbf{0} . \mathbf{9 5}}^{*}$ \\
\hline Block & 86.96 & 11.25 & 7.73 & 1.9 & 2.10 \\
Treatment & 77.29 & 3.08 & 25.1 & 6.2 & 3.14 \\
Within & 134.71 & 33.33 & 4.04 & & \\
Total & 298.96 & & & & \\
\hline
\end{tabular}

*This value from Variance-Gamma package in R-software.

Table 11. ANOMAD for testing equal medians for quality service.

\begin{tabular}{llllcl}
\hline Vari. & SA & Divisor & MAD est. & $\boldsymbol{R}$ & $\boldsymbol{q} \boldsymbol{V G _ { 0 . 9 5 } ^ { * }}$ \\
\hline Block & 94 & 11.25 & 8.35 & 2.60 & 1.93 \\
Treatment & 96 & 3.08 & 31.17 & 9.71 & 2.80 \\
Within & 107 & 33.33 & 3.21 & & \\
Total & 297 & & & & \\
\hline
\end{tabular}

*This value from Variance-Gamma package in R-software.
Effect sizes

Effect sizes (ES) provide another measure of the magnitude of the difference expressed in standard variation units in the original measurement. Thus, with the test of statistical significance and the interpretation of the effect size $(E S)$, the researcher can address issues of both statistical significance and practical importance. The most direct one is

$$
\eta^{2}=\frac{S S_{\text {effect }}}{S S_{\text {Total }}}
$$

where $S S$ is the sum of squares. $\eta^{2}$ measures the proportion of the variation in $Y$ that is associated with membership of the different groups defined by $X . \eta^{2}$ is an uncorrected effect size estimate that estimates the amount of variance explained based on the sample, and not based on the entire 
population. $\omega^{2}$ has been suggested to correct for this bias as

$$
\omega^{2}=\frac{d f_{\text {effect }}\left(M S_{\text {effect }}-M S_{\text {error }}\right)}{S S_{\text {Total }}+M S_{\text {error }}}
$$

See; for example, [3], [4], [18], [2] and [14].

These two measures could be extended to ANOMAD as

$$
\eta_{M A D}=\frac{S A_{\text {effect }}}{S A_{\text {Total }}}
$$

and

$$
\omega_{M A D}=\frac{\text { Divsor }_{\text {effect }}\left(M S A_{\text {effect }}-M S A_{\text {error }}\right)}{S A_{\text {Total }}+M S A_{\text {error }}}
$$

Where $\eta_{M A D}$ measures the proportion of MAD in $Y$ that is associated with membership of the different groups defined by $X$. For the above data, Table 12 gives the computations of these measures.

Table 12. The effect sizes for ANOMAD test.

\begin{tabular}{lllll}
\hline & Using mean & \multicolumn{3}{c}{ Using median } \\
\hline & $\eta_{M A D}$ & $\omega_{M A D}$ & $\eta_{M A D}$ & $\omega_{M A D}$ \\
BLSA & 0.291 & 0.137 & 0.31 & 0.19 \\
TRSA & 0.258 & 0.214 & 0.32 & 0.29 \\
\hline
\end{tabular}

From Table 12, note that ANOMAD for testing equal median explains more variation in dependent variable than ANOMAD for testing equal mean.

\section{Conclusion}

The Laplace distribution provides a good approximation to many applications. In these cases, when the tests of equal means or medians are needed, the ANOMAD will be appropriate. The ANOMAD had a very important property where it had been extended to test for equal medians. Moreover, it had given weights to the data rather than square and that ensured stability in statistical inferences.

The ANOMAD had important information about the shifts in means and medians that studied by fitting variance-gamma distribution to $R_{1}, R_{2}, R_{3}$ and $R_{4}$ and tested for equal means or medians. Also, it offered a very effective way to find out the shifts in means and medians graphically. Actually, the graph is a very strong point if one can obtain the right conclusion from it. Two effect size measures are extended to ANOMAD.

\section{References}

[1] Algina, J., Keselman, H.J., \& Penfield, R.D. “An alternative to Cohen's standardized mean difference effect size: A robust parameter and confidence interval in the two independent group's case" Psychological Methods,10, 317-328 (2005).

[2] Bakeman, R. "Recommended effect size statistics for repeated measures designs", Behaiour Research Methods, 37, 379-384 (2005).
[3] Cohen, J. "Statistical power analysis for the behavioral sciences", (2nd ed.), Hillsdale, NJ: Erlbaum (1988).

[4] Cohen, J. "The earth is round $(\mathrm{p}<.05)$ ", American Psychologist, 49, 997-1003 (1994).

[5] Elamir, E.A.H. "On uses of mean absolute deviation: decomposition, skewness and correlation coefficients", Metron: International Journal of Statistic, LXX, n.2-3, 145-164 (2012).

[6] Gorard S. "Revisiting a 90-year-old debate: the advantages of the mean deviation", British Journal of Educational Studies, 53, 417-430 (2005)

[7] Gorard, S. "Introducing the mean absolute deviation 'effect' size", International Journal of Research \& Method in Education 38(2): 105-114 (2015).

[8] Gradshteyn, I.S., and Ryzhik, I.M., "Table of Integrals, Series, and Products", Academic Press (1980).

[9] Granger, C.W.J. and Z. Ding, "Some properties of absolute return", Annales D'economie et de Statistique, 40, 67-91 (1995).

[10] Haas, M., Mittnik, S. and M.S. Paolella, "Modelling and predicting market risk with Laplace-Gaussian mixture distributions" Applied Financial Economics, 16, 1145-1162 (2006).

[11] Habib, E.A.E "Correlation coefficients based on mean absolute deviation about median" International Journal of Statistics and Systems, 6, 413-428 (2011).

[12] Kotz, S, Kozubowski, T. J., and Podgórski, K. "The Laplace Distribution and Generalizations" Birkhauser, Boston (2001).

[13] Kozubowski,, T. and K. Podgorski, "Asymmetric Laplace laws and modelling financial data" Mathematical and Computer Modelling - special issue, Eds, Mitnik, S., Rachev, S.T.,: Stable non-Gaussian models in finance and econometrics, 34, 1003-1021 (2001).

[14] Lakens, D. "Calculating and reporting effect sizes to facilitate cumulative science: a practical primer for t-tests and ANOVAs" Frontiers in Psychology, 4, 1-12 (2013).

[15] Linden, M., "A model for stock return distribution" International Journal of Finance and Economics, 6, 159-169 (2001).

[16] Mittnik, S., Paolella, M.S. and S.T. Rachev, "Unconditional and conditional distributional models for the Nikkei Index", Asia Pacific Financial Markets, 5, 99-128 (1998).

[17] Neter, J., Kutner, H., Nachtsheim, C. and Wasserman, W. "Applied linear statistical models" 4th ed., McGraw-Hill (1996).

[18] Olejnik,S.,and Algina, "Measures of effect size for comparative studies: applications, interpretations, and limitations" Contemporary Educational Psychology, 25, 241-286 (2000).

[19] Pham-Gia, T. and T. L. Hung "The mean and median absolute deviations" Mathematical and Computer Modeling 34, 921-936 (2001).

[20] Puig, P. and Stephens, M. A. "Tests of fit for the Laplace distribution, with applications" Technometrics 42, 417-424 (2000). 
[21] Sabarinath, A. and A.K. Anilkumar. "Modeling of sunspot numbers by a modified binary mixture of Laplace distribution functions", Solar Physics, 250, 183-197 (2008).

[22] Seneta, E. "Fitting the variance-gamma model to financial data", Journal of Applied Probability. 41A:177-187 (2004).
[23] Srivastava, H.M., Nadarajah, S. and S. Kotz,. "Some generalizations of the Laplace distribution", Applied Mathematics and Computation, 182, 223-231(2006). 